Check for updates

Cite this: Phys. Chem. Chem. Phys., 2017, 19, 29905

Received 7th August 2017, Accepted 13th September 2017

DOI: $10.1039 / c 7 c p 05357 f$

rsc.li/pccp

\title{
Why is surface diffusion the same in ultrastable, ordinary, aged, and ultrathin molecular glasses? $\dagger$
}

\author{
K. L. Ngai, ${ }^{* a}$ Marian Paluch ${ }^{a b}$ and Cristian Rodríguez-Tinoco iD *ab
}

\begin{abstract}
Recently Fakhraai and coworkers measured surface diffusion in ultrastable glass produced by vapor deposition, ordinary glass with and without physical aging, and ultrathin films of the same molecular glassformer, $N, N^{\prime}$-bis(3-methylphenyl)- $N, N^{\prime}$-diphenylbenzidine (TPD). Diffusion on the surfaces of all these glasses is greatly enhanced compared with the bulk diffusion similar to that previously found by others, but remarkably the surface diffusion coefficients $D_{S}$ measured are practically the same. The observed independence of $D_{S}$ from changes of structural $\alpha$-relaxation due to densification or finite-size effect has an impact on the current understanding of the physical origin of enhanced surface diffusion. We have demonstrated before and also here that the primitive relaxation time $\tau_{0}$ of the coupling model, or its analogue $\tau_{\beta}$, the Johari-Goldstein $\beta$-relaxation, can explain quantitatively the enhancement found in ordinary glasses. In this paper, we assemble together considerable experimental evidence to show that the changes in $\tau_{\beta}$ and $\tau_{0}$ of ultrastable glasses, aged ordinary glasses, and ultrathin-films are all insignificant when compared with ordinary glasses. Thus, in the context of the explanation of the enhanced surface diffusion given by the coupling model, these collective experimental facts on $\tau_{\beta}$ and $\tau_{0}$ further explain approximately the same $D_{\mathcal{S}}$ in the different glasses of TPD as found by Fakhraai and coworkers.
\end{abstract}

\section{Introduction}

Surface diffusion coefficients $D_{\mathrm{S}}(T)$ of several molecular glassformers including indomethacin (IMC), nifedipine (NIF), and ortho-terphenyl (OTP), ${ }^{1-3}$ and a canonical metallic glass, $\mathrm{Pd}_{40} \mathrm{Cu}_{30} \mathrm{Ni}_{10} \mathrm{P}_{20}\left(\mathrm{Pd}_{40}\right)^{4}$ were measured at temperatures slightly above and mostly below the bulk glass transition temperature $T_{\mathrm{g}}$ using the method of surface-grating decay. The surface diffusion coefficients $D_{\mathrm{S}}(T)$ are found to be many orders of magnitude larger than the bulk diffusion coefficients $D_{\mathrm{V}}(T)$ at the same temperature. The size of the enhancement of $D_{\mathrm{S}}$ over $D_{\mathrm{V}}$ depends on the glass-former, as shown by nearly the two orders of magnitude greater enhancement in the case of OTP than that in IMC. In all these previous studies ${ }^{1-4}$ at temperatures below $T_{\mathrm{g}}$ the glasses were formed ordinarily by rate cooling or liquid quenching, and are referred to as ordinary glass (OG) to distinguish from a new class of ultrastable glasses (SG) produced by physical vapor deposition. Several theoretical models were proposed to address this huge enhancement of surface mobility of small molecule $\mathrm{OGs}^{5-7}$ and metallic glass. ${ }^{8}$

\footnotetext{
${ }^{a}$ Silesian Center for Education and Interdisciplinary Research,

75 Pulku Piechoty 1, 41-500 Chorzow, Poland. E-mail: kiangai@yahoo.com, cristian.rodriguez-tinoco@smcebi.edu.pl

${ }^{b}$ Institute of Physics, University of Silesia, Uniwersytecka 4, 40-007 Katowice, Poland

$\dagger$ Electronic supplementary information (ESI) available. See DOI: 10.1039/ c7cp05357f
}

Recently Fakhraai and coworkers ${ }^{9-11}$ have investigated the effect of variations in bulk dynamics on the surface diffusion of the molecular glass, $N, N^{\prime}$-bis(3-methylphenyl)- $N, N^{\prime}$-diphenylbenzidine (TPD) with its ordinary $T_{\mathrm{g}}=330 \mathrm{~K}$. Using the tobacco mosaic virus as a probe particle, they measured $D_{\mathrm{S}}(T)$ on glasses of the same composition but with a large difference in bulk relaxation dynamics and in the glass transition temperature. The glasses of TPD include the ordinary glass (OG) obtained by liquid quenching, annealed glass after physical aging at $0.9 T_{\mathrm{g}}$ for a week, ultrastable glass (SG) fabricated by physical vapor deposition at various substrate temperatures, and 12 to $30 \mathrm{~nm}$ thin films. The fictive temperature $T_{\mathrm{f}}$ is reduced in aged glass and much reduced in the SG. The onset temperature for the transformation from the stable glass to the supercooled liquid, $T_{\text {on }}$, is significantly higher than the $T_{\mathrm{g}}$ of the OG. These changes imply orders of magnitude increase of the structural $\alpha$-relaxation time $\tau_{\alpha}$ in the SG. It is generally believed that the high surface mobility or the much larger surface diffusion coefficients $D_{\mathrm{S}}(T)$ rather than $D_{\mathrm{V}}(T)$ plays a critical role in allowing the formation of highly stable glasses. ${ }^{12,13}$ Glasses of higher stability will be formed in systems where surface diffusion is faster. This is borne out by $\mathrm{OTP}^{2}$ having a larger $D_{\mathrm{S}}\left(T_{\mathrm{g}}\right)$ than $\mathrm{IMC}^{1}$ and also having formed a more stable glass than IMC. ${ }^{12,13}$ However, despite the large difference in the bulk dynamics of SG, OG, and annealed OG of TPD, the surface diffusion coefficients of these glasses measured ${ }^{9-11}$ turn out to be nearly identical at two temperatures below the $T_{\mathrm{g}}$ of bulk OG. These results have led 
Fakhraai and coworkers to suggest that surface diffusion has no dependence on the bulk relaxation dynamics when measured below $T_{\mathrm{g}}$, and to question the validity of theories proposed to account for the size of the $D_{\mathrm{S}}(T)$.

Furthermore, Fakhraai and coworkers ${ }^{11}$ measured the average $\alpha$-relaxation dynamics in ultrathin films of molecular glass TPD supported on a silicon substrate with film thickness $h$ in the range $12 \mathrm{~nm}<h<53 \mathrm{~nm}$. As the film thickness is decreased, $T_{\mathrm{g}}$ decreases rapidly, and the average relaxation time of the films is shorter by $\mathbf{6}-\mathbf{1 4}$ orders of magnitude, depending on the measuring temperature below $T_{\mathrm{g}}$ of bulk TPD. The enhancement of relaxation in the TPD thin films also originates from the high mobility of the free surface, which induces faster dynamics of the film interior as concluded from studies of the reduction of the glass transition temperature of polymer thin films. ${ }^{14-20}$ Surface diffusion of the thin films was measured by Fakhraai and coworkers. ${ }^{11}$ Again, surprisingly they found that the surface diffusion coefficients $D_{\mathrm{S}}(T)$ are approximately the same, in spite of the large variations of the averaged film relaxation dynamics for film thicknesses in the range of $12 \mathrm{~nm}<h<400 \mathrm{~nm}$. Even more surprising, the $D_{\mathrm{S}}(T)$ of the thin films are practically the same as that of the SG and OG, indicating a complete decoupling of the average film relaxation and surface diffusion. ${ }^{11}$

Collectively the results from the studies of Fakhraai and coworkers have an impact on the research on surface diffusion, ultrastable glasses, and dynamics of thin films. The results also challenge theories of surface diffusion enhancement, and may yield insight into the $\alpha$-relaxation dynamics of the ultrastable glass, which cannot be directly measured. The basic question is why is the surface diffusion coefficient $D_{\mathrm{S}}(T)$ approximately the same despite widely different structural $\alpha$-relaxation dynamics in the different cases? Part of the answer is at hand if a mechanism for the enhanced $D_{\mathrm{S}}(T)$ can be found and is present in all cases. The answer is complete if the mechanism can give quantitatively the size of the enhanced $D_{\mathrm{S}}(T)$ with approximately the same value for all cases. In this paper, we provide such a mechanism in the primitive relaxation of the coupling model ${ }^{21-25}$ or its analogue the Johari-Goldstein (JG) $\beta$-relaxation ${ }^{25-29}$ exemplified in experiment and simulations. $^{21}$ It has been demonstrated before ${ }^{6,8}$ that the primitive relaxation time $\tau_{0}$ or the JG $\beta$-relaxation time $\tau_{\beta}$ can account for the size of the enhanced $D_{\mathrm{S}}(T)$. In this paper, we show directly from experiments that these relaxation times have approximately the same values in all cases, and thus provide an answer to the question why the measured $D_{\mathrm{S}}(T)$ all have approximately the same value. Moreover, the change of $\tau_{\beta}$ in ordinary glasses at ambient pressure and under high pressures up to $500 \mathrm{MPa}$ is shown to be similar to that found between $\tau_{\beta}$ in SG and $\tau_{\beta}$ in OG. We use this result to support our explanation of the roughly invariant surface diffusion coefficient in SG, OG, and annealed OG as well as in nanometer thin films.

\section{Evidence from experiments}

The basic question from the findings of Fakhraai et al. is why the surface diffusion coefficient $D_{\mathrm{S}}(T)$ is approximately the same in SG, OG, aged OG, and nanometer thin films, despite widely different bulk structural $\alpha$-relaxation dynamics. One way to answer this question is to identify the mechanism that is omnipresent, and can account quantitatively for the size of the surface diffusion enhancement, $D_{\mathrm{S}}(T) / D_{\mathrm{V}}(T)$, in all four cases. But before doing that, one must be able to have a mechanism working in the simpler case of bulk OG before showing it continues to work for the other three more sophisticated cases, particularly the SG. Here we consider the mechanism from the primitive relaxation of the coupling model (CM) and its analogue, the JG $\beta$-relaxation.

\section{A. OG}

In our previous applications of the CM to surface diffusion in $\mathrm{OG},{ }^{6}$ it was assumed at the surface that intermolecular cooperativity of the $\alpha$-relaxation is totally removed and the many-body relaxation is reduced to the primitive relaxation..$^{21-25,28,29}$ Consequently, the $\alpha$-relaxation time at the surface is given by the primitive relaxation time $\tau_{0}(T)$, which can be many orders of magnitude shorter than $\tau_{\alpha}(T)$ in the bulk according to the time-honored CM equation,

$$
\tau_{\alpha}=\left[t_{\mathrm{c}}{ }^{-n} \tau_{0}\right]^{1 /(1-n)},
$$

if $n$ is not zero. In eqn (1) $t_{\mathrm{c}}$ is the onset time of classical $\operatorname{chaos}^{21-24}$ and its magnitude depends on the interaction potential. Its value is $\approx 1$ to $2 \mathrm{ps}$ for soft matter including molecular glass-formers and polymers and was determined by quasielastic neutron scattering experiments and molecular dynamics simulations. ${ }^{21}$ The parameter $(1-n)$ is the fractional exponent of the Kohlrausch correlation function,

$$
\varphi(t)=\exp \left[-\left(t / \tau_{\alpha}\right)^{1-n}\right]
$$

According to the CM, $\tau_{0}$ is the $\alpha$-relaxation time that the structural relaxation would have if all the cooperativity associated with the many-body $\alpha$-relaxation had been removed, and the coupling parameter $n$ is reduced to zero. In fact from eqn (1), it is clear $\tau_{\alpha}$ is reduced to $\tau_{0}$ when $n$ becomes equal to zero. The JG $\beta$-relaxation is an analogue of the primitive relaxation, ${ }^{21,25,28,29}$ and their relaxation times are found in general to be approximately equal,

$$
\tau_{\beta}(T) \approx \tau_{0}(T)
$$

This relation has been verified multiple times in many glass-formers of different types since the first paper in $1998,{ }^{25}$ and is justified by both the primitive relaxation and the JG $\beta$-relaxation being non-cooperative precursors of the structural $\alpha$-relaxation and having similar properties. ${ }^{21}$

Free of neighboring molecules and totally free space to explore on one side, molecules diffusing on the surface are not slowed down by intermolecular coupling, provided there is no widespread hydrogen bonding and extensive penetration of the molecules into the interior to constrain and retard surface diffusion. Thus, at the surface the coupling parameter $n$ can become zero or nearly zero. The $\alpha$-relaxation time, $\tau_{\mathrm{S}}(T)$, at the surface is obtained by substituting $n=0$ into eqn (1), and therefore is the same or nearly the same as $\tau_{0}(T)$,

$$
\tau_{\mathrm{S}}(T)=\tau_{0}(T)
$$


From eqn (4) and the relation of $\tau_{\mathrm{S}}(T)$ to the surface diffusion coefficient, $D_{\mathrm{S}}(T)$, given by $D_{\mathrm{S}}(T)=d^{2} / 4 \tau_{\mathrm{S}}(T)$, the CM immediately predicts $D_{\mathrm{S}}(T)$ quantitatively by

$$
D_{\mathrm{S}}(T)=d^{2} / 4 \tau_{0}(T)
$$

where $d$ is the size of the molecule. Taking relation (3) into consideration, we have

$$
\tau_{\mathrm{S}}(T) \approx \tau_{\beta}(T),
$$

and alternatively, $D_{\mathrm{S}}(T)$ can be determined from the JG $\beta$-relaxation time by

$$
D_{\mathrm{S}}(T) \approx d^{2} / 4 \tau_{\beta}(T)
$$

Predicting $D_{\mathrm{S}}(T)$ by eqn (5) requires calculating the primitive $\tau_{0}(T)$ from eqn (1), which needs the input of $\tau_{\alpha}(T)$ and $n$. By contrast, eqn (7) directly determines $D_{\mathrm{S}}(T)$ from the experimental $\tau_{\beta}(T)$ without performing any calculation. This is worth emphasizing at the outset because we shall apply it to surface diffusion in SG, OG, annealed OG, and thin films later on.

Although eqn (5) and (7) are straightforward, the magnitude of $d$ is not exactly known. This indeterminate parameter is eliminated if the enhancement of surface diffusion given by the ratio, $D_{\mathrm{S}}(T) / D_{\mathrm{V}}(T)$, is considered. Incidentally, the experimental values of this ratio at $T_{\mathrm{g}}$ for several molecular glass-formers are published in the literature. ${ }^{1-4,30}$ If $D_{\mathrm{S}}(T) / D_{\mathrm{V}}(T)$ is the same as the ratio $\tau_{\alpha}(T) / \tau_{\mathrm{S}}(T)$ or $\tau_{\alpha}(T) / \tau_{0}(T)$ except for a factor $3 / 2$ which is insignificant for the consideration herein and henceforth is neglected, then via eqn (1) $D_{\mathrm{S}}(T) / D_{\mathrm{V}}(T)$ is given by

$$
\frac{D_{\mathrm{S}}(T)}{D_{\mathrm{V}}(T)}=\frac{\tau_{\alpha}(T)}{\tau_{\mathrm{s}}(T)}=\frac{\tau_{\alpha}(T)}{\tau_{0}(T)}=\left[\frac{\tau_{\alpha}(T)}{t_{\mathrm{c}}}\right]^{n} .
$$

Furthermore from eqn (6), we have approximately

$$
\frac{D_{\mathrm{S}}(T)}{D_{\mathrm{V}}(T)}=\frac{\tau_{\alpha}(T)}{\tau_{\mathrm{s}}(T)} \approx \frac{\tau_{\alpha}(T)}{\tau_{\beta}(T)} .
$$

For ordinary glasses, the values of $\tau_{\alpha}(T)$ and $n$ are known at temperatures above and below $T_{\mathrm{g}}$ from dielectric relaxation. For indomethacin (IMC), the value of $n=0.41$ was determined. ${ }^{6}$ Using this value together with $t_{\mathrm{c}}=1$ to $2 \mathrm{ps}$ for soft matter and the choice of $\tau_{\alpha}\left(T_{\mathrm{g}}\right)=10^{2}$ or $10^{3} \mathrm{~s}$, the CM eqn (1) gives $\tau_{0}\left(T_{\mathrm{g}}\right)=10^{-3.62}$ and $10^{-3.03} \mathrm{~s}$ respectively. Assuming $d=1 \mathrm{~nm}$, eqn (5) predicts $D_{\mathrm{S}}\left(T_{\mathrm{g}}\right)=10^{-15.16}$ and $10^{-15.75} \mathrm{~m}^{2} \mathrm{~s}^{-1}$ compared to the experimental value of $D_{\mathrm{S}}\left(T_{\mathrm{g}}\right)=10^{-14.3} \mathrm{~m}^{2} \mathrm{~s}^{-1}$ (see inset of Fig. 1). The discrepancy can be due to the arbitrary choice of the value of $d$. On the other hand, after eliminating $d$, eqn (8) yields $\tau_{\alpha}\left(T_{\mathrm{g}}\right) / \tau_{\mathrm{S}}\left(T_{\mathrm{g}}\right) \approx 10^{6} \mathrm{~s}$, which is in order of magnitude agreement with the experimental value of $D_{\mathrm{S}}\left(T_{\mathrm{g}}\right) / D_{\mathrm{v}}\left(T_{\mathrm{g}}\right) .{ }^{1}$ The temperature dependence of $\tau_{0}(T)$ is also about the same as that of the $D_{\mathrm{S}}(T)$ data below $T_{\mathrm{g}}=314 \mathrm{~K}$ of OG, indicating $\tau_{0}(T)$ continues to match $\tau_{\mathrm{S}}(T)$ in value in the OG of IMC. The JG $\beta$-relaxation of IMC is not resolved at ambient pressure (albeit resolved at a high pressure of $400 \mathrm{MPa}$ ), and its relaxation time $\tau_{\beta}(T)$ at ambient pressure cannot be determined exactly.

In this paper we test the $\mathrm{CM}$ further by considering the new case of OTP, which had its JG $\beta$-relaxation resolved in the

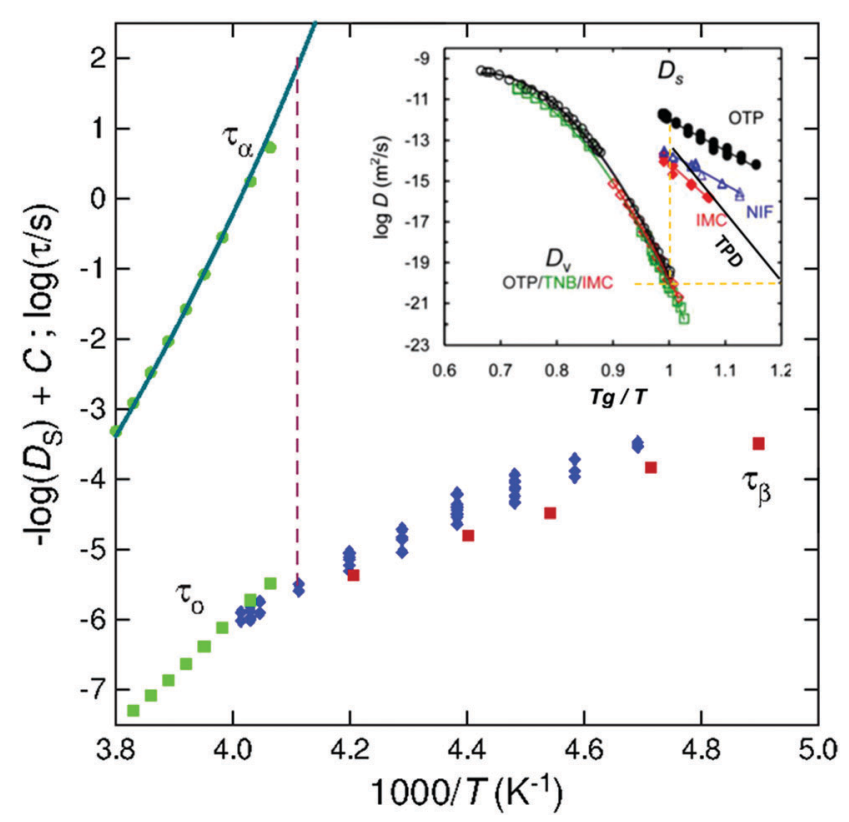

Fig. 1 Green closed circles and squares are the $\alpha$-relaxation times $\tau_{\alpha}(T)$, and the primitive relaxation times $\tau_{0}(T)$ (calculated), and the red closed squares are the $\beta$-relaxation times $\tau_{\beta}(T)$ of bulk OTP. The blue closed diamonds represent $-\log D_{S}(T)+C$ with $C=-17.35$ and the original data of $D_{S}(T)$ are from ref. 2. The inset showing $D_{S}(T)$ and $D_{V}(T)$ is reproduced from ref. 2 . Added is the line, which is a fit of the $D_{S}(T)$ of the OG of TPD by the Arrhenius dependence.

$\mathrm{OG},{ }^{27,31}$ and the experimental values of $\tau_{\beta}(T)$ can be used to directly predict $D_{\mathrm{S}}(T)$ via eqn (7).

OTP has a larger value of $n=0.50$ than IMC at a temperature near $T_{\mathrm{g}}{ }^{32}$ The dielectric $\tau_{\alpha}(T)$ and a Vogel-Fulcher fit are shown in Fig. 1 together with $\tau_{\beta}(T)$, the calculated $\tau_{0}(T)$, and $D_{\mathrm{S}}(T)$ after it was shifted by a constant to match roughly $\tau_{0}(T)$ above $T_{\mathrm{g}}$. Although $\tau_{0}(T)$ can only be obtained above $T_{\mathrm{g}}$, $\tau_{\beta}(T)$ was determined experimentally below $T_{\mathrm{g}}$. By extrapolating the Arrhenius temperature dependence of $\tau_{\beta}(T)$ back to $T_{\mathrm{g}}$ the relationship $\tau_{\beta}(T) \approx \tau_{0}(T)$ is verified. Hence, from eqn (8) and (9), we have at $T_{\mathrm{g}}$. the CM predicted value of

$$
D_{\mathrm{S}}\left(T_{\mathrm{g}}\right) / D_{\mathrm{V}}\left(T_{\mathrm{g}}\right)=\tau_{\alpha}\left(T_{\mathrm{g}}\right) / \tau_{\mathrm{S}}\left(T_{\mathrm{g}}\right)=\tau_{\alpha}\left(T_{\mathrm{g}}\right) / \tau_{0}\left(T_{\mathrm{g}}\right)=10^{7.5},
$$

and

$$
D_{\mathrm{S}}\left(T_{\mathrm{g}}\right) / D_{\mathrm{V}}\left(T_{\mathrm{g}}\right)=\tau_{\alpha}\left(T_{\mathrm{g}}\right) / \tau_{\mathrm{S}}\left(T_{\mathrm{g}}\right) \approx \tau_{\alpha}\left(T_{\mathrm{g}}\right) / \tau_{\beta}\left(T_{\mathrm{g}}\right) \approx 10^{7.5}
$$

This prediction of enhanced surface mobility based on $\tau_{0}(T)$ and $\tau_{\beta}(T)$ is to be compared with the experimental value of $D_{\mathrm{S}}\left(T_{\mathrm{g}}\right) / D_{\mathrm{V}}\left(T_{\mathrm{g}}\right) \approx 10^{7.6}$ at $T_{\mathrm{g}}$ shown in the inset of Fig. 1 taken from the paper of Zhang et al. ${ }^{2}$ Deeper into the glassy state, the shifted $D_{\mathrm{S}}(T)$ and $\tau_{\beta}(T)$ are within half a decade apart and can be considered the same if the uncertainty of $\tau_{\beta}(T)$ determined from the broad frequency dispersion of the JG $\beta$-relaxation is taken into consideration. Thus eqn (8) and (9) for $T \leq T_{\mathrm{g}}$ in OG of OTP is verified.

We can obtain $D_{\mathrm{S}}\left(T_{\mathrm{g}}\right)$ of OTP directly from the experimental value of $\tau_{\beta}\left(T_{\mathrm{g}}\right)$ by using eqn (7) and again assuming $d=1 \mathrm{~nm}$. The Arrhenius extrapolation of $\tau_{\beta}(T)$ in Fig. 1 determines 
$\tau_{\beta}\left(T_{\mathrm{g}}\right)=10^{-5.56} \mathrm{~s}$. Hence the predicted value of $D_{\mathrm{S}}\left(T_{\mathrm{g}}\right)$ is $10^{-13.22} \mathrm{~m}^{2} \mathrm{~s}^{-1}$, which is smaller than the experimental value of $10^{-12.1} \mathrm{~m}^{2} \mathrm{~s}^{-1}$. Assumed in eqn (5) and (7) is that $\tau_{\mathrm{s}}\left(T_{\mathrm{g}}\right)$ is the same as the CM primitive relaxation time $\tau_{0}\left(T_{\mathrm{g}}\right) \approx \tau_{\beta}\left(T_{\mathrm{g}}\right)$ in the OTP bulk. But the actual value of $\tau_{0}\left(T_{\mathrm{g}}\right) \approx \tau_{\beta}\left(T_{\mathrm{g}}\right)$ at the surface can be shorter, due to more free space, and hence the actual value of $D_{\mathrm{S}}\left(T_{\mathrm{g}}\right)=10^{-13.22} \mathrm{~m}^{2} \mathrm{~s}^{-1}$ calculated by eqn (7) can underestimate the real enhancement. It can be seen from Fig. 1 that $\tau_{\beta}(T)$ continues to describe well the temperature dependence of $D_{\mathrm{S}}(T)$ deep into the glassy state of OTP. This means the $D_{\mathrm{S}}(T)$ from eqn (7) not only agrees with the experimental value at $T_{\mathrm{g}}$ but also at temperatures below it.

The exercise presented in the above in the case of OTP demonstrates that the experimental data of $\tau_{\beta}(T)$ can be used directly via eqn (7) to determine $D_{\mathrm{S}}(T)$. Therefore, if there is no or little change in $\tau_{\beta}(T)$ in glasses of different origins, we can immediate conclude that the surface diffusion coefficients $D_{\mathrm{S}}(T)$ measured in these glasses will be practically the same. This point is relevant for understanding the collection of studies of the surface diffusion of SG, OG, and annealed OG of TPD in the following subsections B and C.

Shown in the inset of Fig. 1 is the Arrhenius fit of the measured tracer surface diffusion coefficients of the OG of TPD. ${ }^{10}$ The enhancement $D_{\mathrm{S}}\left(T_{\mathrm{g}}\right) / D_{\mathrm{V}}\left(T_{\mathrm{g}}\right)$ of TPD at $T_{\mathrm{g}}$ lies in between that of the surface self-diffusion of IMC and OTP. If the tracer surface diffusion of TPD measured is not too different from self-diffusion, the approximately same order of magnitude of the enhancement at $T_{\mathrm{g}}$ of TPD and OTP can be understood from the comparable value of $n=0.50$ and 0.48 for TPD and OTP respectively.

\section{B. Aged OG}

From the previous Section A we have validated eqn (6) and (7) and established that the JG $\beta$-relaxation time $\tau_{\beta}(T)$ is a quantitative indicator of the enhanced surface diffusion coefficient $D_{\mathrm{S}}(T)$ for $T \leq T_{\mathrm{g}}$ in the OG. Now, if the OG is densified by physical aging over a long period of time, any change of $D_{\mathrm{S}}(T)$ will be observed by the corresponding change in $\tau_{\beta}(T)$. It is known from the classical study of physical aging of $43.3 \mathrm{~mol} \%$ toluene in pyridine glass at several temperatures ${ }^{33}$ that there is practically no change of the $\beta$ loss peak frequency, $f_{\beta}=\left(1 / 2 \pi \tau_{\beta}\right)$, except a reduction of the amplitude of the loss. A slight increase of the peak frequency was found on aging the JG $\beta$-relaxation of glassy polyvinylethylene. ${ }^{34}$ On the other hand, a small increase of peak frequency was found on aging of dipropyleneglycol dibenzoate. ${ }^{35}$ In all cases, including others not mentioned, there is either no change or a small change of $\tau_{\beta}$ by physical aging. From this general aging property of $\tau_{\beta}(T)$, it follows from eqn (6) and (7) that $\tau_{\mathrm{S}}(T)$ and $D_{\mathrm{S}}(T)$ of the aged TPD glass are nearly the same as that of OG, as found by Fakhraai and coworkers.

\section{SG}

The key for continued use of eqn (6) and (7) to predict the enhancement of surface diffusion at $T_{\mathrm{g}}$ or $T_{\mathrm{on}}$ in SG is to have data on $\tau_{\beta}(T)$ of SG. The onset temperature $T_{\text {on }}$ is defined as the intersection of the extrapolated glassy line and the tangent of the transformation from the glassy state to the supercooled liquid state. Among the SGs produced by vapor deposition, only the SG of toluene ${ }^{36,37}$ has been studied by dielectric relaxation and reported. ${ }^{38}$ Toluene is a rigid molecule and its sole secondary relaxation is the JG $\beta$-relaxation. ${ }^{28}$ The relationship between the $\alpha$ - and the $\beta$-relaxations of toluene is in accord with the CM eqn (1)-(3) as shown by an example in the inset of Fig. 2. Shown there is the reported dielectric loss spectrum of toluene at $119 \mathrm{~K}$ with $f_{\alpha}=0.2 \mathrm{~Hz}^{39}$ and the Kohlrausch fit with exponent $(1-n)=0.52$. The calculated primitive frequency $f_{0}=\left(1 / 2 \pi \tau_{0}\right)$ is about half a decade higher than $f_{\beta}=\left(1 / 2 \pi \tau_{\beta}\right)$, verifying the approximate eqn (3).

The experimental data of $\tau_{\beta}(T)$ of a toluene SG deposited at a substrate temperature $98 \mathrm{~K}\left(=0.84 T_{\mathrm{g}}\right)$ from $\mathrm{Yu}$ et $a{ }^{38}$ are reproduced in Fig. 2. The value of $T_{\text {on }}$ of the SG is $123 \mathrm{~K},{ }^{36}$ and the corresponding $1000 / T_{\text {on }}$ is located in Fig. 2 by the vertical black broken line. The other vertical broken line is at $1000 / T_{\mathrm{g}}$ with $T_{\mathrm{g}}=117 \mathrm{~K}$. Shown also are $\tau_{\beta}(T)$ and $\tau_{\alpha}(T)$ of the toluene OG from dielectric and NMR measurements. ${ }^{40,41}$ Remarkably, $\tau_{\beta}(T)$ of the SG is longer than the OG by less than a decade at the same temperature, and the $T$-dependence of both are Arrhenius with the activation energies $E_{\beta}=27 \pm 3$ and $25 \pm 2 \mathrm{~kJ} \mathrm{~mol}^{-1}$ respectively. From these experimental facts, eqn (6) and (7) of the CM readily explain why the surface diffusion time $\tau_{\mathrm{S}}(T)$ and coefficient $D_{\mathrm{S}}(T)$ of the SG are practically the same as those of the OG.

We have extrapolated the Arrhenius $T$-dependence of $\tau_{\beta}(T)$ in the SG to higher temperatures to intersect $1000 / T_{\text {on }}$. The intersection determines $\tau_{\beta}\left(T_{\text {on }}\right)=10^{-4.31} \mathrm{~S}$ for the SG, while a similar

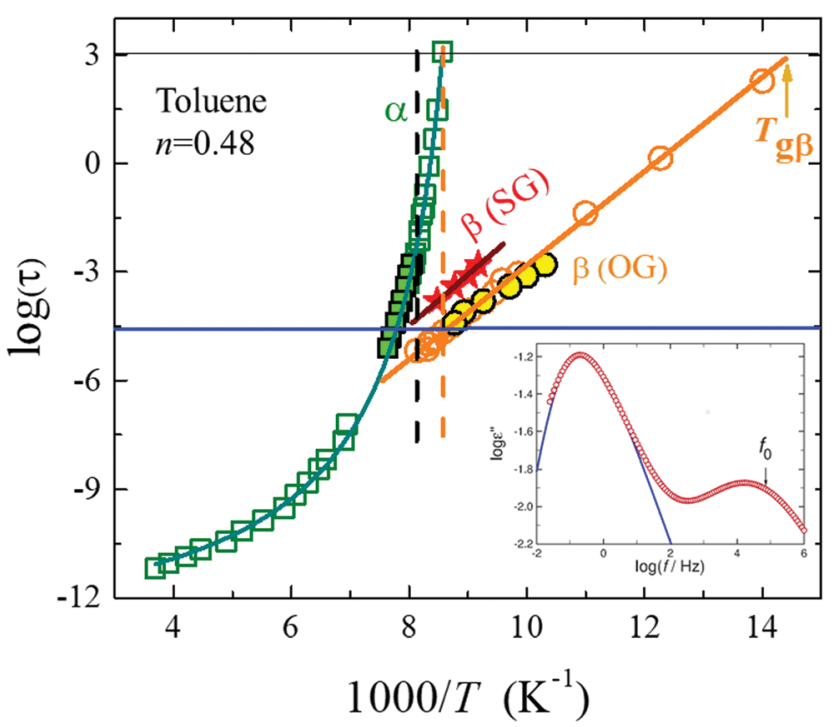

Fig. 2 The main figure is reproduced from Yu et al. ${ }^{38}$ It shows logarithmic relaxation times of $\alpha$ and $\beta$ processes of ordinary glass (open symbols), and the relaxation times for the vapor-deposited samples (solid symbols) vs. reciprocal temperature. Added are the two vertical lines located at $1000 / T_{\mathrm{g}}$

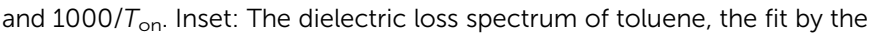
Fourier transform of the Kohlrausch function with $n=0.48$, and the calculated value of the primitive frequency $f_{0}$. 
operation determines $\tau_{\beta}\left(T_{\mathrm{g}}\right)=10^{-4.57} \mathrm{~S}$ for the OG. The nearly same value of $\tau_{\beta}$ suggests also $\tau_{\mathrm{S}}(T)$ of the SG at $T_{\mathrm{on}}$ is similar to $\tau_{\mathrm{S}}(T)$ of the OG at $T_{\mathrm{g}}$.

\section{Nanometer thin films}

Zhang and Fakhraai ${ }^{11}$ measured average $\alpha$-relaxation time in ultrathin films of molecular glass TPD supported on a silicon substrate with film thicknesses ranging from $12 \mathrm{~nm}$ to $400 \mathrm{~nm}$. The film $T_{\mathrm{g}}$ decreases rapidly with decreasing film thickness. They also measured the tracer surface diffusion coefficient $D_{\mathrm{S}}(T)$ of all the films and found that the $D_{\mathrm{S}}(T)$ are all enhanced at all temperatures by the same orders of magnitudes as the $D_{\mathrm{S}}(T)$ of $\mathrm{OG}$ and $\mathrm{SG}$, despite the fact that the average film $\alpha$-relaxation time decreases significantly with decreasing film thickness.

It is generally agreed that the enhanced surface mobility in supported or freestanding polymer thin films is primarily the cause of the decrease of the film $T_{\mathrm{g}}$ as thickness is decreased. The enhanced surface mobility with the fast relaxation time $\tau_{\mathrm{S}}(T)$ is transmitted into the interior of the film but the effect is attenuated layer by layer. ${ }^{19}$ The substrate may have an opposite effect on the mobility if physical or chemical interaction with the film exists at the interface. Consequently, the averaged $\alpha$-relaxation of the film measured is slower than at the surface, and the effective relaxation time is longer than $\tau_{\mathrm{S}}(T)$ as in the study of Zhang and Fakhraai. However, the JG $\beta$-relaxation is a local process and its relaxation time $\tau_{\beta}(T)$ is not sensitive to changes of film thickness, as is supported by experiments. For example, dielectric measurements of poly(methyl methacrylate) (PMMA) thin films show little change of $\tau_{\beta}(T)$ for thickness down to $20 \mathrm{~nm}$. The $\tau_{\beta}(T)$ of the $15 \mathrm{~nm}$, and $9.5 \mathrm{~nm}$ thick films are only about a factor of 5 and 8 respectively shorter than the thick $1070 \mathrm{~nm}$ film, while the change of $T_{\mathrm{g}}$ is more than $20 \mathrm{~K}^{42}$ Direct measurement of surface relaxation of syndiotactic (PMMA) films by lateral force microscopy also finds the $\tau_{\beta}(T)$ at the surface shorter than the bulk by about 1 decade at higher temperatures of measurements. ${ }^{43}$ Thus, from eqn (6) and (7), the small difference between $\tau_{\beta}(T)$ in thin films and in bulk explains the same for the surface diffusion $D_{\mathrm{S}}(T)$ observed in glassy thin films of TPD compared to OG.

It is worthwhile mentioning a related experimental fact of the average segmental relaxation time, $\tau_{\alpha}^{\text {nano }}$, measured by dielectric relaxation on a $40 \mathrm{~nm}$, and by PCS on a $22 \mathrm{~nm}$ freestanding polystyrene (PS) thin film. The values of $\tau_{\alpha}^{\text {nano }}(T)$ were shown to match the primitive relaxation times $\tau_{0}(T)$ in the glassy state of bulk PS. ${ }^{44}$ In these ultrathin freestanding films the high mobility of the two free surfaces dominate in determining $\tau_{\alpha}^{\text {nano }}$, and hence the finding of $\tau_{\alpha}^{\text {nano }}(T) \approx \tau_{0}(T)$ supports the fact that $\tau_{\mathrm{S}}(T)$ is the same as $\tau_{0}(T)$, or eqn (4).

The cooperative $\alpha$-relaxation in nominal glass-formers such as OTP at 10 degrees above $T_{\mathrm{g}}$ has a length-scale of the order of $20 \mathrm{~nm}$ already. ${ }^{45}$ Therefore, when the film thickness is much reduced to the order of $1 \mathrm{~nm}$, not only the surface mobility dominates the entire film but also the interior is also removed of the cooperativity provided that the substrate has no effect. The ideal situation is realized in the $1.5 \mathrm{~nm}$ thin films of poly(methylphenylsiloxane) confined in galleys of nanocomposites $^{19,46}$ or poly(dimethylsiloxane) confined in $2 \mathrm{~nm}$ glass pores, ${ }^{47}$ and the calculated $\tau_{0}(T)$ by eqn (1) from the bulk is in quantitative agreement with the experimental $\tau_{\mathrm{S}}(T)$ as shown in ref. 19 (see Fig. S1 for the $1.5 \mathrm{~nm}$ thin film in the ESI $\dagger$ ). Thus eqn (4) is verified and, via eqn (5), it supports the explanation of the nearly no change of $D_{\mathrm{S}}(T)$ to change of film thickness.

\section{Comparing SG at ambient pressure with OG at elevated pressures}

Studies of the structure of vapor-deposited SG have shown the differences from OG, which include higher density, different local packing and orientations of the molecules. ${ }^{48,49}$ Since higher density is a primary cause of the stability of SG, it is profitable to consider the glasses formed by applying very high pressure to the liquid and comparing some properties. This task is relevant in view of a recent experiment, ${ }^{50}$ which compares the pressure dependence of the onset of devitrification, $T_{\text {on }}$, between the SG and OG of IMC. The dynamics and thermodynamic stabilities of the two glasses are very different at ambient pressure. Ultrastable glasses of IMC have densities up to $1.4 \%$ more than the liquid-cooled glass. ${ }^{13,37}$ The two glasses have different $\mathrm{d} T_{\text {on }} / \mathrm{d} P$ values at low pressures, but become nearly the same when $T_{\text {on }}$ of the OG approaches that of the SG with increase of pressure to $300 \mathrm{MPa} .^{50}$

At about $75 \mathrm{MPa}$, the increase of $T_{\text {on }}$ of OG with pressure has caught up with the $T_{\text {on }}$ of SG at ambient pressure. From this, we can expect at higher pressures, such as a few hundred MPa routinely reached in dielectric studies of the dynamics of glass-formers, that $T_{\text {on }}$ as well as the density of the pressurized OG is significantly higher than the UG at ambient pressure. Thus, the difference in $\tau_{\beta}(T)$ between the pressurized OG and OG at ambient pressure in the glass state should be larger than that between the SG and OG at ambient pressure. This can be verified by examining some experimental $\tau_{\beta}(T)$ data of OG at ambient and elevated pressure such as poly(phenylglycidylether) (PPGE) ${ }^{51}$ diglycidyl ether of bisphenol-A with $M_{\mathrm{w}}=380 \mathrm{~g} \mathrm{~mol}^{-1}$ (also known as EPON 828), ${ }^{52}$ and di-propylene glycol dibenzoate (DiPGDB). ${ }^{35}$ In Fig. S2-S4 (ESI $\dagger$ ) are the data of $\tau_{\beta}$ and $\tau_{\alpha}$ of the three glass-formers shown at a fixed temperature as a function of pressure, and at a fixed pressure as a function of temperature. From these plots, $T_{\mathrm{g}}$ changes by $54 \mathrm{~K}$ for EPON 828 with the increase in pressure of $400 \mathrm{MPa}$; by $43 \mathrm{~K}$ and $77 \mathrm{~K}$ for DiPGDB with increase of 268 and $530 \mathrm{MPa}$ respectively; and by 17 and $64 \mathrm{~K}$ for PPGE with increase of 95.5 and $500 \mathrm{MPa}$ respectively. The $T_{\mathrm{g}}$ change of $17 \mathrm{~K}$ at $95.5 \mathrm{MPa}$ matches the difference between $T_{\mathrm{g}}=242.3 \mathrm{~K}$ and $T_{\mathrm{on}}=259 \mathrm{~K}$ for the most stable glass of OTP vapor-deposited at $0.85 T_{\mathrm{g}}{ }^{12}$ Even for the much larger increase of pressure up to $500 \mathrm{MPa}$, it only changes $\tau_{\beta}$ by one to two orders of magnitude in the glassy state at a fixed temperature in all cases. The change of $\tau_{\beta}$ is even less at pressures lower than $100 \mathrm{MPa}$ to match the increase in density with that of the IMC SG at ambient pressure. This we can use as corroborative evidence to support the small difference in $\tau_{\beta}$ 
between SG and OG at ambient pressure, and the use of the CM eqn (6) and (7) to explain the same surface diffusion coefficient for the two glasses. The properties of pressurized OG and SG produced by vapor deposition are the same as long as the pressure on the former is maintained, and the common cause is significant densification of the glass. By no means do the similar properties diminish the achievement of vapor-deposited SG in producing glassy materials with properties very different from OG at ambient pressure. By contrast, when the pressure is released, the pressurized glass returns to OG. Nevertheless, the physics of the pressurized glass is the same as SG as far as the comparison is confined to their glassy states. The analogy is no longer valid when the relationship of the glassy state to the equilibrium liquid is considered. For example, during transformation of the SG, the emerging liquid phase is at ambient pressure, and cannot be compared to the transformation of pressurized OG, where both the glass and liquid phases are under high pressure. Consequently, SG and pressurized OG do not exhibit the same transformation behavior. More importantly, the limiting fictive temperature $\left(T_{\mathrm{f}}^{\prime}\right)$ of a SG is lower than the OG, while the $T_{\mathrm{f}}^{\prime}$ of the pressurized OG is higher than that of the OG at ambient pressure (note that this does not contradict the higher density of the pressurized OG than the ambient pressure OG). To be more specific, when calculating the $T_{\mathrm{f}}^{\prime}$ of a SG, one considers the corresponding liquid at ambient pressure, while the $T_{\mathrm{f}}^{\prime}$ of the pressurized OG refers to the liquid at the corresponding pressure. Again, since the definition of $T_{\mathrm{f}}^{\prime}$ of either glass involves the corresponding equilibrium liquid, the mentioned analogy is not applicable in this point.

It is worthwhile to point out from the data shown in Fig. S2-S4 $(\mathrm{ESI} \dagger)$ that $\tau_{\beta}(T, P)$ has approximately the same value at any arbitrary chosen long time $\tau_{\alpha}(T, P)$, independent of the choice of the fixed $T$ as a function of $P$, or the choice of fixed $P$ as a function of $T \cdot{ }^{35,51,52}$ The relationship $\tau_{\beta}(T, P) \approx \tau_{0}(T, P)$ has also been verified.

\section{Conclusions}

The findings of practically no change of the surface diffusion coefficient $D_{\mathrm{S}}(T)$ on ultrastable glass (SG), ordinary glass (OG) with and without physical aging, and nano-meter glassy thin films of TPD by Fakhraai and coworkers are interesting, and certainly have an impact on identifying the mechanism of enhanced surface diffusion. They suggest that fast surface diffusion is decoupled from the bulk dynamics, although the exact mechanism of this decoupling merits further investigation. We show the mechanism of decoupling is the removal of intermolecular coupling or cooperativity in relaxation and diffusion of molecules at the surface, resulting in the surface relaxation time $\tau_{\mathrm{S}}(T)$ being the same as the primitive relaxation time $\tau_{0}(T)$ of the coupling model (CM), and orders of magnitude shorter than the bulk $\alpha$-relaxation time $\tau_{\alpha}(T)$. The enhanced surface diffusion is given by $D_{\mathrm{S}}(T)=d^{2} / 4 \tau_{\mathrm{S}}(T)=d^{2} / 4 \tau_{0}(T)$ where $d$ is the size of the molecule. In the case of OG at $T_{\mathrm{g}}$, the values of $\tau_{\alpha}\left(T_{\mathrm{g}}\right)$ and the coupling parameter $n$ are known, and the calculated $D_{\mathrm{S}}\left(T_{\mathrm{g}}\right)$ are in semi-quantitative agreement such as in the case of IMC, ${ }^{4}$ OTP herein, and ethylcyclohexane (ECH). ${ }^{53}$ $D_{\mathrm{S}}\left(T_{\mathrm{g}}\right)$ of ECH is several orders of magnitude less enhanced than that of IMC and OTP, ${ }^{53}$ but it is still consistent with the CM eqn (8) because ECH has a smaller $n \leq 0.32$ than IMC and OTP. From the fact that the Johari-Goldstein $\beta$-relaxation is the analogue of the primitive relaxation, and its relaxation time $\tau_{\beta}(T)$ is approximately the same as $\tau_{0}(T)$ as proven in many materials, one can take the values of $\tau_{\beta}(T)$ from experiment and directly calculate the enhanced surface diffusion in the glassy state by $D_{\mathrm{S}}(T) \approx d^{2} / 4 \tau_{\beta}(T)$. The crux of our explanation of the approximate invariance of $D_{\mathrm{S}}(T)$ in SG, OG with and without physical aging, and nano-meter glassy thin films of TPD is the corresponding approximate invariance of $\tau_{\beta}(T)$, despite the huge differences in the bulk $\alpha$-dynamics. This property of $\tau_{\beta}(T)$ is demonstrated directly from experimental data in all these different glasses. It is perhaps unsurprising since both the primitive relaxation and the JG $\beta$-relaxation are local process without cooperativity, although a more in-depth study to substantiate it theoretically is needed and will be forthcoming. We compare also the change of $\tau_{\beta}(T)$ in some other ordinary glasses by elevating the pressure up to $500 \mathrm{MPa}$. The advantage of studying OG under pressure is because $\tau_{\beta}(T)$ is resolved in the glassy state of many glass-formers. Pressure below $100 \mathrm{MPa}$ is able to densify the OG to the same level and have a similar $T_{\text {on }}$ as the best SG deposited at $0.85 T_{\mathrm{g}}$. The change of $\tau_{\beta}(T)$ in the pressurized OG is found to be small. Much higher $T_{\text {on }}$ is found in OG pressurized at higher levels above $100 \mathrm{MPa}$. Notwithstanding, the change of $\tau_{\beta}(T)$ is still not large. This comparative study of $\tau_{\beta}(T)$ in OG at elevated pressures lends support to the explanation for the ultrastable glass.

In this paper we use eqn (5)-(7) exclusively and directly from the experimental $\tau_{\beta}$ or the theoretical $\tau_{0}$ to compare with the experimental value of $D_{\mathrm{S}}(T)$ of SG like TPD. The CM eqn (1) need not be used to calculate $\tau_{0}$ from the structural $\alpha$-relaxation time $\tau_{\alpha}$. The exception is the previous work on indomethacin (IMC) ${ }^{6}$ where $\tau_{\alpha}$ is known from experiment above and below $T_{\mathrm{g}}$, and OTP above $T_{\mathrm{g}}$ in Fig. 1 herein. This situation in IMC and OTP confers the benefit of predicting the size of the enhancement by the ratio, $\tau_{\alpha} / \tau_{0}$, and comparing with the experimental value of $D_{\mathrm{S}}(T) / D_{\mathrm{V}}(T)$, as successfully done for IMC and OTP. In the case of SG prepared by vapor deposition, $\tau_{\alpha}$ is not known and eqn (1) cannot and need not be used to calculate $\tau_{0}$ or $\tau_{\beta}$ in the stable glass.

\section{Conflicts of interest}

There are no conflicts of interest to declare.

\section{Acknowledgements}

We thank Prof. Ranko Richert and Prof. Haibin Yu for sharing with us their data on the secondary relaxation in the ultrastable glass of toluene. C. R.-T. acknowledges support from the National Science Centre through the Polonez scheme (Grant No. DEC-2015/19/P/ST3/03540/2). This project has received 
funding from the European Union's Horizon 2020 research and innovation programme under the Marie Skłodowska-Curie grant agreement no. 665778 .

\section{References}

1 L. Zhu, C. W. Brian, S. F. Swallen, P. T. Straus, M. D. Ediger and L. Yu, Surface Self-Diffusion of an Organic Glass, Phys. Rev. Lett., 2011, 106, 256103.

2 W. Zhang, C. W. Brian and L. Yu, Fast Surface Diffusion of Amorphous $o$-Terphenyl and Its Competition with Viscous Flow in Surface Evolution, J. Phys. Chem. B, 2015, 119, 5071-5078.

3 C. W. Brian and L. Yu, Surface Self-Diffusion of Organic Glasses, J. Phys. Chem. A, 2013, 117, 13303-13309.

4 C. R. Cao, Y. M. Lu, H. Y. Bai and W. H. Wang, High surface mobility and fast surface enhanced crystallization of metallic glass, Appl. Phys. Lett., 2015, 107, 141606.

5 J. D. Stevenson and P. G. Wolynes, On the surface of glasses, J. Chem. Phys., 2008, 129, 234514.

6 S. Capaccioli, K. L. Ngai, M. Paluch and D. Prevosto, Mechanism of fast surface self-diffusion of an organic glass, Phys. Rev. E: Stat., Nonlinear, Soft Matter Phys., 2012, 86, 51503.

7 S. Mirigian and K. S. Schweizer, Theory of activated glassy relaxation, mobility gradients, surface diffusion, and vitrification in free standing thin films, J. Chem. Phys., 2015, 143, 244705.

8 K. L. Ngai, S. Capaccioli, C. R. Cao, H. Y. Bai and W. H. Wang, Quantitative explanation of the enhancement of surface mobility of the metallic glass $\mathrm{Pd}_{40} \mathrm{Cu}_{30} \mathrm{Ni}_{10} \mathrm{P}_{20}$ by the coupling model, J. Non-Cryst. Solids, 2017, 463, 85-89.

9 Y. Zhang, R. Potter, W. Zhang and Z. Fakhraai, Using tobacco mosaic virus to probe enhanced surface diffusion of molecular glasses, Soft Matter, 2016, 12, 9115-9120.

10 Y. Zhang and Z. Fakhraai, Invariant Fast Diffusion on the Surfaces of Ultrastable and Aged Molecular Glasses, Phys. Rev. Lett., 2017, 118, 66101.

11 Y. Zhang and Z. Fakhraai, Decoupling of surface diffusion and relaxation dynamics of molecular glasses, Proc. Natl. Acad. Sci. U. S. A., 2017, 114, 4915-4919.

12 K. R. Whitaker, M. Tylinski, M. Ahrenberg, C. Schick and M. D. Ediger, Kinetic stability and heat capacity of vapor-deposited glasses of $o$-terphenyl, J. Chem. Phys., 2015, 143, 84511.

13 K. J. Dawson, K. L. Kearns, L. Yu, W. Steffen and M. D. Ediger, Physical vapor deposition as a route to hidden amorphous states, Proc. Natl. Acad. Sci. U. S. A., 2009, 106, 15165-15170.

14 J. L. Keddie, R. A. L. Jones and R. A. Cory, Size-Dependent Depression of the Glass Transition Temperature in Polymer Films, Europhys. Lett., 1994, 27, 59-64.

15 K. Tanaka, A. Takahara and T. Kajiyama, Rheological Analysis of Surface Relaxation Process of Monodisperse Polystyrene Films, Macromolecules, 2000, 33, 7588-7593.

16 Z. Fakhraai and J. A. Forrest, Measuring the surface dynamics of glassy polymers, Science, 2008, 319, 600-604.

17 T. W. Kim and K.-S. Choi, Nanoporous $\mathrm{BiVO}_{4}$ Photoanodes with Dual-Layer Oxygen Evolution Catalysts for Solar Water Splitting, Science, 2014, 343, 990-994.
18 Z. Yang, Y. Fujii, F. K. Lee, C.-H. Lam and O. K. C. Tsui, Glass Transition Dynamics and Surface Layer Mobility in Unentangled Polystyrene Films, Science, 2010, 328, 1676-1679.

19 K. L. Ngai, Interpreting the dynamics of nano-confined glass-formers and thin polymer films: importance of starting from a viable theory for the bulk, J. Polym. Sci., Part B: Polym. Phys., 2006, 44, 2980-2995.

20 M. Alcoutlabi and G. B. McKenna, Effects of confinement on material behaviour at the nanometre size scale, J. Phys.: Condens. Matter, 2005, 17, R461-R524.

21 K. L. Ngai, Relaxation and Diffusion in Complex Systems, Springer New York, New York, NY, 2011.

22 K. Y. Tsang and K. L. Ngai, Relaxation in interacting arrays of oscillators, Phys. Rev. E: Stat. Phys., Plasmas, Fluids, Relat. Interdiscip. Top., 1996, 54, R3067-R3070.

23 K. Y. Tsang and K. L. Ngai, Dynamics of relaxing systems subjected to nonlinear interactions, Phys. Rev. E: Stat. Phys., Plasmas, Fluids, Relat. Interdiscip. Top., 1997, 56, R17-R20.

24 K. L. Ngai and K. Y. Tsang, Similarity of relaxation in supercooled liquids and interacting arrays of oscillators, Phys. Rev. E: Stat. Phys., Plasmas, Fluids, Relat. Interdiscip. Top., 1999, 60, 4511-4517.

25 K. L. Ngai, Relation between some secondary relaxations and the $\alpha$ relaxations in glass-forming materials according to the coupling model, J. Chem. Phys., 1998, 109, 6982-6994.

26 G. P. Johari and M. Goldstein, Molecular mobility in simple glasses, J. Phys. Chem., 1970, 74, 2034-2035.

27 G. P. Johari and M. Goldstein, Viscous Liquids and the Glass Transition. II. Secondary Relaxations in Glasses of Rigid Molecules, J. Chem. Phys., 1970, 53, 2372-2388.

28 K. L. Ngai and M. Paluch, Classification of secondary relaxation in glass-formers based on dynamic properties, J. Chem. Phys., 2004, 120, 857-873.

29 K. L. Ngai, An extended coupling model description of the evolution of dynamics with time in supercooled liquids and ionic conductors, J. Phys.: Condens. Matter, 2003, 15, S1107-S1125.

30 S. Ruan, W. Zhang, Y. Sun, M. D. Ediger and L. Yu, Surface diffusion and surface crystal growth of tris-naphthyl benzene glasses, J. Chem. Phys., 2016, 145, 64503.

$31 \mathrm{H}$. Fujimori and M. Oguni, Correlation index and activation energy ratio as parameters characterizing the structure of liquid and glass, Solid State Commun., 1995, 94, 157-162.

32 C. León and K. L. Ngai, Rapidity of the Change of the Kohlrausch Exponent of the $\alpha$-Relaxation of Glass-Forming Liquids at $T_{\mathrm{B}}$ or $T_{\beta}$ and Consequences, J. Phys. Chem. B, 1999, 103, 4045-4051.

33 G. P. Johari, Effect of annealing on the secondary relaxations in glasses, J. Chem. Phys., 1982, 77, 4619-4626.

34 R. Casalini and C. M. Roland, Aging of the secondary relaxation to probe structural relaxation in the glassy state, Phys. Rev. Lett., 2009, 102, 35701.

35 D. Prevosto, S. Capaccioli, M. Lucchesi, P. A. Rolla and K. L. Ngai, Does the entropy and volume dependence of the structural $\alpha$-relaxation originate from the Johari-Goldstein $\beta$-relaxation?, J. Non-Cryst. Solids, 2009, 355, 705-711. 
36 M. Ahrenberg, Y. Z. Chua, K. R. Whitaker, H. Huth, M. D. Ediger and C. Schick, In situ investigation of vapordeposited glasses of toluene and ethylbenzene via alternating current chip-nanocalorimetry, J. Chem. Phys., 2013, 138, 24501.

37 E. Leon-Gutierrez, A. Sepúlveda, G. Garcia, M. T. ClavagueraMora, J. Rodríguez-Viejo, T. Matsuo, L. H. Allen, T. L. Yu and S. Satija, Stability of thin film glasses of toluene and ethylbenzene formed by vapor deposition: an in situ nanocalorimetric study, Phys. Chem. Chem. Phys., 2010, 12, 14693-14698.

38 H. B. Yu, M. Tylinski, A. Guiseppi-Elie, M. D. Ediger and R. Richert, Suppression of $\beta$ Relaxation in Vapor-Deposited Ultrastable Glasses, Phys. Rev. Lett., 2015, 115, 185501.

39 X. Li, M. Wang, R. Liu, K. L. Ngai, Y. Tian, L.-M. Wang and S. Capaccioli, Secondary relaxation dynamics in rigid glassforming molecular liquids with related structures, J. Chem. Phys., 2015, 143, 104505.

40 A. Kudlik, C. Tschirwitz, S. Benkhof, T. Blochowicz and E. Rössler, Slow secondary relaxation process in supercooled liquids, Europhys. Lett., 1997, 40, 649-654.

41 E. Rössler and H. Sillescu, $2 \mathrm{H}$ NMR Study of supercooled toluene, Chem. Phys. Lett., 1984, 112, 94-98.

42 K. Fukao, S. Uno, Y. Miyamoto, A. Hoshino and H. Miyaji, Dynamics of $\alpha$ and $\beta$ processes in thin polymer films: poly(vinyl acetate) and poly(methyl methacrylate), Phys. Rev. E: Stat., Nonlinear, Soft Matter Phys., 2001, 64, 51807.

43 K. Akabori, K. Tanaka, T. Nagamura, A. Takahara and T. Kajiyama, Molecular Motion in Ultrathin Polystyrene Films: Dynamic Mechanical Analysis of Surface and Interfacial Effects, Macromolecules, 2005, 38, 9735-9741.

44 K. L. Ngai, S. Capaccioli, M. Paluch and D. Prevosto, Temperature dependence of the structural relaxation time in equilibrium below the nominal $T_{\mathrm{g}}$ : results from freestanding polymer films, J. Phys. Chem. B, 2014, 118, 5608-5614.
45 S. A. Reinsberg, A. Heuer, B. Doliwa, H. Zimmermann and H. W. Spiess, Comparative study of the NMR length scale of dynamic heterogeneities of three different glass formers, J. Non-Cryst. Solids, 2002, 307-310, 208-214.

46 S. H. Anastasiadis, K. Karatasos, G. Vlachos, E. Manias and E. P. Giannelis, Nanoscopic-Confinement Effects on Local Dynamics, Phys. Rev. Lett., 2000, 84, 915-918.

47 A. Schönhals, H. Goering, C. Schick, B. Frick and R. Zorn, Polymers in nanoconfinement: What can be learned from relaxation and scattering experiments?, J. Non-Cryst. Solids, 2005, 351, 2668-2677.

48 S. F. Swallen, K. L. Kearns, M. K. Mapes, Y. S. Kim, R. J. McMahon, M. D. Ediger, T. Wu, L. Yu and S. Satija, Organic glasses with exceptional thermodynamic and kinetic stability, Science, 2007, 315, 353-356.

49 A. Gujral, K. A. O'Hara, M. F. Toney, M. L. Chabinyc and M. D. Ediger, Structural Characterization of Vapor-Deposited Glasses of an Organic Hole Transport Material with X-ray Scattering, Chem. Mater., 2015, 27, 3341-3348.

50 C. Rodríguez-Tinoco, M. González-Silveira, M. Barrio, P. Lloveras, J. L. Tamarit, J.-L. Garden and J. RodríguezViejo, Ultrastable glasses portray similar behaviour to ordinary glasses at high pressure, Sci. Rep., 2016, 6, 34296.

51 D. Prevosto, S. Capaccioli, S. Sharifi, K. Kessairi, M. Lucchesi and P. A. Rolla, Secondary dynamics in glass formers: relation with the structural dynamics and the glass transition, J. Non-Cryst. Solids, 2007, 353, 4278-4282.

52 K. L. Ngai, J. Habasaki, D. Prevosto, S. Capaccioli and M. Paluch, Thermodynamic scaling of $\alpha$-relaxation time and viscosity stems from the Johari-Goldstein $\beta$-relaxation or the primitive relaxation of the coupling model, J. Chem. Phys., 2012, 137, 34511.

53 K. L. Ngai and S. Capaccioli, Comment on the paper entitled Limited surface mobility inhibits stable glass formation for 2-ethyl-1-hexanol, published in J. Chem. Phys., 2017, 146, 203317, arXiv:1706.00874 [cond-mat.soft]. 